\title{
12 tips to teaching (legal and ethical aspects of) research ethics/responsible conduct of research
}

\author{
Abstract \\ Teaching research ethics is a requirement within modern health science, nursing and \\ medical curricula. We have drawn on our experience of designing, developing and \\ integrating the teaching of research ethics in a new, fully integrated medical school \\ curriculum, delivered using Problem Based Learning (PBL) and the recent literature relating \\ to the teaching of research ethics to produce the following 12 Top Tips designed to \\ encourage readers to seek opportunities to embed this teaching within a variety of curricula.
}

\section{Introduction}

All healthcare workers including doctors practicing in a modern, evidence based culture need to have an understanding of both the methodological validity and the ethics that underpins good research. The guidelines on research ethics are a recent development within the UK; it wasn't until the1990's that a uniform, national system of research ethics committees (COREC, now NRES) evolved. However even with the implementation of research ethics committees to oversee and ensure the ethical conduct of research in the health, clinical and social sciences, examples of unethical research still occur.

Unfortunately, the impact of unethical research has far reaching consequences. Recent examples of the media reporting of the controversies that surround Andrew Wakefield and the MMR vaccine have illustrated that public confidence in medical research is damaged by such adverse incidents. In the case of Andrew Wakefield and his research into the possible link between the development of autism and the use of the Measles, Mumps and Rubella vaccine (MMR), the consequences of unethical research have been far reaching and long lasting. The reporting of this research in the established, high impact and well respected and trusted medical journal the Lancet in 1999 (Wakefield et al., 1999), led to a dramatic fall in the uptake of the MMR vaccine with the result that the incidence of cases of measles has increased. Uptake of the MMR vaccine remains compromised even though subsequent, robust medical research has failed to find a link between incidence of autism and the MMR vaccine. More than 12 years after this controversial research was published in the Lancet, the original paper was finally retracted in 2010 . This retraction was as a direct result of the publication of the General Medical Council (GMC) Fitness to Practice Report for Andrew Wakefield stating that the conduct of the research had not been undertaken in an ethically responsible manner (General Medical Council, 2010a). . As a further consequence of this Fitness to Practice Report, Andrew Wakefield was struck off the British medical register by the GMC after being found guilty of serious professional misconduct This ruling clearly indicates that the GMC recognises the importance of conducting ethical, robust research and has developed clear guidelines to all registered doctors who undertake and conduct research (General Medical Council, 2010b, General Medical Council, 2006). These guidelines state that all research should be based on a protocol that has been developed according to the good practice guidance given by government and other research and professional bodies. In addition all protocols should seek and obtain approval from a Research Ethics Committee (REC).

Within the United Kingdom (UK) the Tomorrows Doctors 2009 (General Medical Council, 2009) provides a specific blueprint for undergraduate medical curricula within the UK and it sets out specific leaning outcomes that the GMC expects medical schools to deliver. In order to meet the need for modern doctors to understand the importance of undertaking, ethically governed research the GMC has established that an understanding of research ethics is a specific outcome in undergraduate medical curricula in the UK. It clearly states that not only should a graduate be expected to apply the scientific method to medical 
research but they should also "understand the ethical and governance issues involved in medical research" (General Medical Council, 2009 p 18).

Although it is recognised that there is a need for teaching research ethics (termed responsible conduct of research in USA) (Eisen and Berry, 2002), evidence presented by Lehmann et al indicate that in Canadian and US medical schools the teaching of medical ethics including research ethics is varied both in content and in pedagogical methods (Lehmann et al., 2004). They surveyed 91 medical schools and found that only $54 \%$ covered the areas of research ethics and $42 \%$ covered the role of ethics committees. Information about the programmes that teach research ethics mainly describe teaching at postgraduate level; there is limited literature discussing undergraduate teaching.(Steneck and Bulger, 2007). In addition Heitman et al demonstrated that the level of knowledge of research ethics of graduate students in a group of American universities was inadequate (Heitman et al., 2007a) and furthermore that graduate students in biomedical science or health professions only scored " marginally higher" than new undergraduates suggesting that effective training was lacking.

There is a large body of published papers on research ethics that outlines the lengthy, and time consuming requirement and bureaucracy that is associated with the process of obtaining ethical approval for undertaking research (Jamrozik, 2004, Wald, 2004, Glasziou and Chalmers, 2004). Understandably, this can lead to unwillingness to engage with the formal process and the paradigms which drive it. It is also worth recognising that there is a significant proportion of health, medical and bioscience researchers who were educated at a time before serious consideration of the ethics of research was common practice.

Similarly this may also be the case for some of the most senior educators in medical schools. Nonetheless, at the present time the value given to undertaking ethical research has been recognised and is reflected in Tomorrow's Doctors 2009(General Medical Council, 2009), that outlines the necessity of equipping students with a detailed understanding of the process, the value and the relevance of research ethics within taught undergraduate medical curricula.

It should also be recognised that today's students will graduate into a world where advances in medical and scientific knowledge and understanding is occurring at an unparalleled rate. For example new technologies and knowledge that have underpinned research into stem cells and the human genome project have raised ethical questions and issues and these seem set to continue as science and medicine continues to advance. These new technologies and emerging treatments have a corresponding financial implication. It is important to recognise that there is clearly an overlap between commercial interests and the development and practice of medicine within society. This overlap has the potential to raise conflicts of interest when the role of the financial support offered by industry has to be balanced with the practice of responsible and ethical medicine. Influential and respected journals such as Science, Nature, the British Medical Journal and the Lancet have adopted common standards for the review process and promote the publishing of research that has been ethically undertaken and has scientific integrity. In addition financial disclosure of authors is encouraged.

Developing future cohorts of students who understand value and practice ethical research may result in a future where research ethics review is viewed in a less contentious way by future researchers. Additional positive impacts may include the associated reduction in cost and delays associated with poor submissions to REC's as well as the restoration of public confidence in the ethical nature of medical research. It is important that students develop an awareness of the technologies and medical treatments that are continuing to emerge and that they are equipped to contend with the ethical implications that these developments will bring. Similarly it is important that we recognise that the government funding of our scientific 
and health research has purported that research decisions are accountable to a public that has a vested interest in research decisions.

We recognise that simply advising new students that ethical behaviour is a necessity in a modern environment of medical research and evidence based practice is not sufficient. Students will benefit from learning the background, issues, benefits, concepts, theory and skills associated with research ethics. We have drawn on our experience of designing, developing and integrating the teaching of research ethics in a new, fully integrated medical school curriculum, delivered using Problem Based Learning (PBL) and the recent literature relating to the teaching of research ethics to produce the following 12 Top Tips.

Tip 1; The Learning EnvironmentA key requirement is to undertake the teaching of research ethics within a positive and supportive learning environment. It is essential that the School or Department has a learning philosophy that supports the teaching of research ethics within the curriculum. This philosophy should emanate from the Dean and the Course Director, and this philosophy should be reflected by the faculty and colleagues who value and support research ethics teaching. This view is identified by Lehmann et al (Lehmann et al., 2004)who found that the 123 Deans of US and Canadian Medical schools, unanimously agreed that role models could have a significant effect on a student's ethical behaviour." (Miles et al., 1989)In addition this should manifest itself as administrative and financial support for this teaching.(Kalichman, 2007a)

In order for students and staff to be able to engage with the issues involved in ethical research it is important that a 'Safe space for active involvement with the ethical issues' is provided. This Safe space should include an open and trustful environment, where staff and students are both emotionally supportive and facilitating towards the issues. There should be an understanding and an acceptance that students and staff will have different educational backgrounds and areas of knowledge but that sharing of information may be valuable and beneficial to all students and staff.(Hafferty and Franks, 1994a) (Heitman et al., 2007b, Eisen and Berry, 2002)

At an organisational level there should be a clear message that the issues of morality and ethics including research ethics are ubiquitous to the day to day role of health care practitioners and that there is a routine requirement for ethics within the medical, health sciences and the scientific community(Hafferty and Franks, 1994a). This message should be apparent to students in the day to day behaviour of faculty who undertake scientific and clinical work.

Tip 2; Students should understand why they need to study research ethics There is international recognition of the need for students to learn about the ethical dilemmas in research. The GMC has established a set of guidelines detailing the core teaching expected within an undergraduate medical degree programme(General Medical Council, 2009), In 1999 a report from the Association of American Medical Colleges (AAMC) suggested that all medical schools must ensure that before graduation students will have demonstrated..."knowledge of...ethical dilemmas in medicine" (Lehmann et al., 2004, Association of American Medical Colleges, 1998). None the less we recognise that there is potential for a curriculum to become information overloaded with respect to the amount of information and skills that a student is expected to know by the time they graduate. Therefore it is important that students understand and value why the ethical and governance issues involved in medical and health science research should be taught as an 
integral part of an undergraduate curriculum. Opportunities should be provided for students to develop an awareness of both the positive view that ethical research is the only acceptable standard, as well as the negative consequences of unethical practice even if that unethical practice is unintentional. To facilitate this, a curricula should

- provide opportunities for students to recognise the critical role research has in underpinning the evidence base of clinical practice. However at the same time provide opportunities for students to develop an awareness of the public's expectation that this research is conducted in an ethically acceptable way. For example, interesting case studies can be used to highlight how easy it is to develop an unethical approach 'if one is not both knowledgeable and vigilant' and the subsequent consequences (General Medical Council, 2010a))

- discuss the equal importance placed on ethical understanding as on factual knowledge and clinical skills publications and guidelines for medical education(General Medical Council, 2009, General Medical Council, 2010b, General Medical Council, 2006, Association of American Medical Colleges, 1998, The Royal College of Physicians and Surgeons of Canada, 2005).

- teach students to recognise that the primary literature used as teaching material reflects the importance of ethical research. Almost all journals have a publishing policy that requires research ethics to have been considered and applied on all publications. (Informa Healthcare, 2010)

- emphasise the importance of undertaking research in an ethical manner and explain that unethical practice may be a criminal matter with significant penalties.

\section{Tip 3; Start early and integrate throughout course}

We recognise and understand that the process of learning and understanding ethics including research ethics will evolve and grow as students gain knowledge and appreciation of both research and clinical dilemmas and we suggest that teaching research ethics is undertaken at the earliest opportunity. However, Heitman et al 2007 and Powell 2007 (Heitman et al., 2007b, Powell et al., 2007) suggest that teaching research ethics delivered as a one off course early in a curriculum is probably insufficient to impart even core concepts and standards in lasting ways.

Therefore in order to successfully embed research ethics teaching it is desirable to integrate it both horizontally and vertically throughout a medical school curriculum(Lehmann et al., 2004). Although we suggest starting research ethics teaching at the earliest opportunity, it is important to recognise that students may come to the course with different attitudes, and levels of knowledge and understanding towards research ethics and that it may be useful to establish this baseline before undertaking teaching (Heitman et al., 2007b). Other reasons include

- Avoiding negative perception of research ethics: In a study of students undertaking doctoral level research with no previous training in research ethics McGee et al showed that students with prior experience of applying for ethical approval were more resistant to training in research ethics (responsible conduct of research) compared to students without prior experience of applying for ethical approval (McGee et al., 2008). They state that 'if what they [the learners] hear is new or does not challenge their thinking in other ways it is relatively easy to incorporate new knowledge into their frame of reference. If what they hear challenges or is in opposition to their prior knowledge they have acquired, the "learning" is more difficult'. Although this finding may reflect the experience of gaining ethical approval for research it is clear that this experience prior to research ethics training has a negative effect on subsequent learning. Therefore it seems appropriate to introduce 
research ethics training into undergraduate curricula for the health care professions at the earliest opportunity. This may ensure that potential future researchers are taught about research ethics with an open frame of mind. Acquiring this knowledge at an early stage in a future researcher's career may also result in a more successful and less negative experience of applying for research ethics approval.

- Avoiding the use of jargon: Another aspect to consider when undertaking ethical research is the need to gain informed consent from research participants. Providing participants with well written, informative participant information sheets is an important aspect of gaining this consent and jargon should be avoided. Students should be encouraged to write and produce such documents before they have adopted professional jargon into their usual communication style. Delivering information to a potential research participant can also be used as an example in communication training.

- Encountering prejudice: Finally it is important that we recognise that students will encounter and receive advice from members of faculty who will challenge the need for research ethics and the acceptability of the review process. Similarly it is important to recognise that students will encounter medical culture outside of the curriculum based timetable; this culture or "hidden curriculum can be antithetical to the goals and content of those courses formally offered"(Hafferty and Franks, $1994 b$ (page 865), Abdulla, 2008). Providing students with an understanding of the arguments that underpin the requirements for research ethics before they are exposed to the counter argument is desirable.

\section{Tip 4: Establish the course outcomes and structure}

It is important to have learning outcomes that are clear, can be seen to have demonstrable value (justifying the time you are requesting to deliver the teaching) can be mapped onto the curriculum, fulfil the requirements of the curriculum and reflect the ethos of the programme. Within the literature there are many documented suggestions for achievable learning objectives for research ethics teaching (Steneck, 2007, Sponholz, 2000, Bulger and Heitman, 2007, Eisen and Berry, 2002, Kalichman and Plemmons, 2007, Plemmons and Kalichman, 2007).

- We suggest there is a value in producing a document that provides:

- clear learning outcomes

- a clear outline that details how learning outcomes can be mapped successfully onto the curriculum.

- information detailing how the learning outcomes are to be delivered within the curriculum. This should include details of teaching sessions associated with their delivery.

- Details of the teaching staff (faculty) required for successful delivery of the teaching

- Clear description of assessment methods and dates, and the requirement for marking of scripts if needed

- The document should be made available to the school's curriculum design and development team, external examiners and appropriate regulatory bodies.

- The learning outcomes should, as much as possible reflect the style and content of other aspects of the curriculum. Ideally they should demonstrate how they link with other teaching and learning opportunities delivered within the medical school (eg Ethical consent in research is an example of how consent must be fully informed linking with general medical ethics and communication skills).

- A lead for research ethics teaching should be identified who can 
- answer questions about this aspect of the curriculum from other faculty members.

- provide advice to other faculty members on teaching research ethics as and when required

- coordinate the delivery of teaching

- be prepared to remain up to date with developments in the field of research ethics teaching and to update the research ethics curriculum as and when appropriate as appropriate

- encourage all members of staff delivering research ethics teaching need to support the need for its inclusion in the undergraduate curriculum and be prepared to challenge colleagues who doubt its value.

\section{Tip 5: Where possible integrate with other aspects of teaching and learning}

Developing a programme or research ethics education into a taught programme will be more successful if it is viewed as an integrated part of the course and not as an additional educational requirement. This will also counter the obstacles of "lack of curriculum time, the limited availability of qualified teachers and the lack of time in faculty schedules" identified by Lehman et al 2004.(Lehmann et al., 2004(page 686), Abdulla, 2008) It will also facilitate the development of a research ethics programme that is both longitudinal and horizontally integrated(Lehmann et al., 2004).

Most new information is easier to understand, learn and retain if it can be linked to other aspects of knowledge familiar to students. In order to locate areas of the curriculum where research ethics can be linked effectively, arrange meetings to discuss with colleagues collectively and individually to spot possible areas for integration and shared teaching. Examples of possible areas that should be considered include

- Health economics; NICE guidance is based on evidence from published literature. It has to be ethical and the debate about the justification for offering or withholding treatment may be used to illustrate research ethics as well as decision making

- Communications skills; communication of information in participant information sheets is of prime importance in research ethics

- Medical statistics; most quantitative papers have a statistical basis, understanding how an under, or over, powered study may be unethical is a teaching tool available to both ethicists and statisticians

- Psychology and social medicine; quality of life is an important concept in medicine, assessment is a valuable research tool but may provide opportunities to discuss research ethics.

- Clinical practice; there is a clear link with general medical ethics and the approach to treatment choices, communication, consent and patient involvement.

- Basic science; basic science research is increasingly dependent on human tissue and analysis of genes and of the genetic basis of disease. The requirement for consent and the potential adverse effects on patients of knowledge of genetic risk may is complex and should be clearly explained and understood. There are also opportunities to detail and outline the professional norms that underpin the integrity of research practice and research discovery.

- Training courses in Research methods, particularly those including the critical reviews of published work are an ideal opportunity to explore the research ethics that underpin the research described. Students can be asked to look carefully at the methodology described and identify flaws in the ethical approach of research studies 
- Finally the Research project: we are aware that some medical curricula still offer students the opportunity to undertake a research project. This can often provide the perfect opportunity to link medical ethics teaching to other areas within a curriculum. However we recognise that current research governance requirements including the obligation to obtaining ethical approval, rather than promoting students to actively obtain research experience are

- emphasising the difficulties of negotiating the bureaucracy of the process and

- encouraging the development of suitable strategies to eliminate or circumvent the requirements for obtaining appropriate approvals(Robinson et al., 2007).

\section{Tip 6: Course material used to deliver research ethics teaching}

Maintaining medical student interest in a subject that may appear to have little, immediate clinical impact can be achieved if a wide variety of different teaching materials are offered and used.

- Teaching material can be delivered in didactic lectures to introduce basic paradigms however the information should be clear and concentrate on understanding of concepts not acquisition of factual knowledge

- Providing links to guidelines from outside bodies allows students to extend their reading and develop their understanding and familiarity with the need for and process of gaining approval. Guidance from University research offices, the operating procedures of NRES committees and research funders such as the MRC ESRC, HTA, BPS in the UK and the FDA and Institutional review boards in the US provide detailed information often with examples of good and bad practice(Kalichman, 2007b)

- Real life clinical examples or case studies that illustrate how, why and when research ethics has had an important influence on practice, or, conversely researchers have failed to produce an "ethical" study resulting in a serious impact on participant welfare, public health, individual practitioners and the profession at large (eg Andrew Wakefield, Northwick Park - Te General), can actively capture students interest(Sponholz, 2000)

- Provide links to online application forms where research ethics approval is required or has to be considered. This should help students to understand ethical issues which they may not have thought of. An example is the information that should be included in a participant information sheet, and the information requested on grant applications or application forms for ethical consent.

- Provide a reading list of valuable original papers and books on the subject. Useful guidance can be obtain from book reviews and recommendations, but we suggest that all recommended course material should be reviewed carefully; writing by philosophers can be challenging to a student who is not fully immersed in the basics of academic philosophy.

- Consider using virtual learning environments (VLE) to allow students to revisit problem areas and work at their own pace. The ideas in research ethics are complex, and some students may find them difficult to grasp. Using a VLE with, for example pod casts of lectures, worked examples research ethics problems supported by explanation allows the student to address areas they find difficult and develop a more comprehensive understanding. 
- Supplement teaching material with self assessment tasks which students can use to confirm their understanding, identify weaknesses and monitor their progression.

Tip 7: Consider using active teaching and group discussion.

There is a clear emphasis within the literature that teaching research ethics can be facilitated by encouraging students to undertake active learning. Providing opportunities for students to listen to alternative view points, discuss their own viewpoints, undertake reasoning and apply ethical reasoning to complex situations.(Sponholz, 2000, McGee et al., 2008, Eisen and Berry, 2002) At the XXXXXXX, we have provided students with the opportunity to take an active learning approach to research ethics based on a piece of their own work. Our research ethics teaching is embedded in a broader research module in which students design a research project and write a research protocol. Students review each other's protocols and discuss the ethical issues associated with the research in a group session. Students are also asked to undertake the protocol review using a form similar to those used by a NHS REC to guide them on the issues that should be considered. In order for these sessions to run successfully it is important that they are facilitated by a tutor with suitable experience such as a REC member. This type of session has several benefits:

- Students are introduced to the concept of a research ethics committee and are exposed to a similar format and process

- They can engender lively debate as students can "defend" their own design and explain their thinking. Equally they are exposed to different viewpoints and concerns that they may need to consider in their own research protocol.

- All group members will often join in bringing different ideas on the problem, possible solutions, and importantly they are also given the opportunity to apply this new knowledge to their own research protocol designs.

- These sessions provide students with an opportunity to apply their theoretical knowledge to a new situation and scenario encouraging deeper learning.

Tip 8: Use Alternative and Varied Sources of Expertise

Research ethics is a multi disciplinary field and it is important to involve the expertise and experience of lawyers, philosophers, ethicists and educationalists and the public. There will be many faculties that have teachers who can provide essential, informed teaching in research ethics additional sources of expertise should be considered as an alternative source of information and experience to support student's learning and understanding of research ethics. At the University of East Anglia we have enlisted the support from members of the University and local NRES Research ethics committees. Interestingly the feedback that we have had from ethics committee members who have contribute to the teaching is that they have found it to be both a useful and a rewarding experience. We have also worked with volunteer members of the public through the Public and Patient Involvement in Research group (PPiRES), a group of trained and informed individuals who actively participate with researchers in the organisation and delivery of research studies. They bring the perspective of the patient and the participant who are engaged in the research process. Their perspective towards them process of research ethics is able to provide a unique and invaluable point of view that is appreciated by the students. Finally consider sharing resources with other schools and departments who may have alternative and complementary expertise.

Tip 9; Additional Learning Opportunities 
Seek addition opportunities out with the course and the core curriculum to expose students to Research ethics instruction even if this is infrequent and minimal. Opportunities that could be considered include

- inviting students to attend guest lectures that bring an interdisciplinary approach to understanding and appreciating research ethics.

- encouraging students to attend less formal arenas such as public lectures where diverse audiences may expose alternative ethical views.

- facilitate students to form discussion groups and journal clubs where research ethics are a discussion point or indeed a main focus.

\section{Tip 10; Offer training and support to a broad based faculty not only research ethics teachers}

It is important to recognise that the attitudes students will develop towards research ethics will be influenced by every teacher that they meet throughout their course and not just the faculty members who deliver the research ethic component of the curriculum. These teachers include members of faculty, clinicians in primary and secondary care, patients and members of the public and the media. As stories about issues of medical research in the media often have an ethical dimension, we recognise there is a significant amount of influence that has an impact on student's behaviours and beliefs within the 'hidden Curriculum'(Hafferty and Franks, 1994b). However there is also plenty of opportunity within the taught curriculum to provide students with a positive learning environment towards research ethics and its role within the curriculum and future practice. To ensure that this occurs, it is really important that all teachers, including those who deliver research ethics teaching, are offered training These training programmes could include

- courses that teach clinicians how to design and run an ethical research study. Good Clinical Practice guidelines were developed by the European Medicines agency as a model for the ethical approach to interventional research in health care settings. Completion of suitable training is required for all researchers in many UK hospitals(European Medicines Agency, 2002).

- an overview of the content, timing and delivery and an understanding of the methods of research ethics teaching within the curriculum. Teachers should be encouraged to deliver an informed view on research ethics concepts and issues. It is important to acknowledge that students learning can be compromised by teachers who express views on research ethics that are misinformed.

- a description of and explanation for the strategies used to assess student's knowledge and understanding of research ethics.

- training on how to distinguish the different types of evidence based practice including various types of research study and design, the audit process, service evaluation and method development. Teachers need to understand when it is important that ethical approval is required before commencing a study irrespective of whether a medical school teaches research ethics as a separate component within their research methods teaching.

- all teachers involved in the delivery of research ethics teaching should be offered continual sources of support and advice. This is particularly relevant as both the bureaucracy and the underlying philosophy of research ethics are constantly evolving.

- courses for faculty members within the basic and clinical sciences that illustrate how ethics including research ethics can relate "to science and medicine at a macro level". This can be encouraged through faculty organised seminars, journal clubs to 
encourage a "routine and everyday place for ethics within the scientific and medical communities"(McGee et al., 2008)

- courses and workshops that provide research ethics training to graduates, post docs and junior faculty members.

- appropriate courses in other schools or institutions.

\section{Tip11; Develop an effective assessment strategy}

Assessing students understanding of research ethics can provide challenges. It is clearly not as easy to assess as other parts of the core curriculum, and there is a danger of over concentrating assessment on the mechanics of applying and achieving ethical approval from ethics committees rather than the understanding of the paradigms of research ethics. We feel that these challenges may be met using a variety of assessment methods at different stages of the course. For example

- it is reported that some courses that teach research ethics assess a pass as full attendance at all sessions with the "premise that more was gained by in -class participation(McGee et al., 2008)"

- undertaking a critical analysis of published research paper is a method of assessment in medical school courses. This can provide an introduction to and consideration of the ethical issues underpinning the research if students are asked to address this within the assessment.

- Inclusion in end of session exam papers is also an option. Short answer questions (SAQs) allow assessment of the students understanding of the principles underlying research ethics. Extended matching questions can be used to assess students' knowledge and understanding of the mechanisms and the process of the ethical review of research, the important legal issues of research, the types of study that do not require ethical review, for instance clinical audit.

- If a student is expected to write or analyse a research protocol as part of their medical course,this could include a research ethics section and the necessary documentation such as participant information sheets and consent forms. These can then be assessed as part of the submitted work.

- The process of ethical review in an active learning session where students undertake critical assessment and ethical argument through role playing, active discussion of case studies or research protocols can provide the basis of a written work which can be submitted for assessment. The student can submit a summary of the session and are assessed on their understanding of the range of ethical issues that have to be considered, and their approach to common problem areas.

- Peer assessment can also be a useful tool. This can take place within the active learning session but it can also be used in other ways for example in the assessment of research protocols. Students can ethically review each other's research protocols. This can provide a level of formative assessment that benefits both the student who reviews a research protocol and the author of the research protocol.

- We suggest that the markers should be selected from faculty members who have demonstrated a proper understanding of research ethics and if possible have sat as members of research ethics committees. Marking schemes with clear marking criteria should be provided to all markers. For all pieces of submitted work written by students we have found that anonymous, single marking with moderation is an effective approach that provides consistency of marking. It is important to ensure that all failures, border fails and a proportion of their remainder are reviewed. 
Tip 12; Evaluate and adapt

It is important that the teaching of research ethics within a curriculum is evaluated and reviewed. We suggest this is necessary to ensure that

- there is consistency in teaching across each cohort and through each successive cohort.

- to ensure that the teaching of research ethics continues to map successfully to current guidance on good practice such as Tomorrow's Doctors 2009.

- to highlight examples of good practice and areas of teaching that have worked well but also to provide details and information about areas where teaching could be adapted, improved or reconsidered.

There are different ways to evaluate an educational programme; these include obtaining questionnaires, written feedback or verbal feedback. However it is important that any feedback received from students is acknowledged and is seen to be acted upon. Encouraging tutors and teachers involved in the teaching of research ethics to act as a critical friend is a useful way to obtain information about areas of teaching that are working well but also areas that need to be adapted or improved. It should also be recognised that these teachers and tutors can be an excellent source of positive advice and useful suggestions. In addition, research ethics teaching can be evaluated by looking at other sources of evidence such as the assessment data associated with the teaching of research ethics, the improvement in writing of research protocols and the process of critically analysing research papers. Finally formal review and evaluation can be provided by external examiners, the process of course review and GMC visits (if appropriate).

\section{Conclusions}

Embedding the teaching of research ethics into a medical curriculum can be challenging. However it is important to consider tailoring this teaching to suit each individual curriculum. Opportunities to include research ethics in current teaching should be sought where ever possible. By successfully providing a positive and supportive environment towards research ethics students will begin to develop knowledge and an understanding of both the methodological validity and the ethics that underpins good research. This should lead to doctors who are aware of the value and necessity of evidence based medicine that will enhance the practice of evidence based medicine and could help to improve the public perception of medical research.

\section{Notes on authors}

LAURA BOWATER is a Senior Lecturer in the School of Medicine, Health Policy and Practice at the University of East Anglia and a panel member of the Faculty of Health University Ethics Committee.

MARK WILKINSON is a Consultant Pathologist at the Norfolk and Norwich University Hospital, Chair of the Faculty of Health University Ethics Committee and an Honorary Senior Lecturer in the School of Medicine, Health Policy and Practice at the University of East Anglia

\section{Acknowledgements}

We would like to thank professor Sam Leinster for providing useful information and help in the writing of this manuscript. 


\section{References}

Lancet retracts MMR paper after GMC finds Andrew Wakefield guilty of dishonesty. British Medical Journal, 281.

ABDULLA, A. (2008) A critical analysis of mini peer assessment tool (mini-PAT). J R Soc Med, 101, 22-6.

ASSOCIATION OF AMERICAN MEDICAL COLLEGES (1998) learning Objectives for Medical Schools.

BULGER, R. E. \& HEITMAN, E. (2007) Expanding Responsible Conduct of Research Instruction across the University. Academic Medicine, 82, 876-879.

EISEN, A. \& BERRY, R. M. (2002) The absent professor: why we don't teach research ethics and what to do about it. Am J Bioeth, 2, 38-49.

EUROPEAN MEDICINES AGENCY (2002) Note for Guidance on Good Clinical Practice,

. London,.

GENERAL MEDICAL COUNCIL (2006) Good Medical Practice.

GENERAL MEDICAL COUNCIL (2009) Tomorrow's Doctors 2009. General Medical Council.

GENERAL MEDICAL COUNCIL (2010a) FITNESS TO PRACTISE PANEL HEARING for Dr Andrew Jeremy WAKEFIELD

GMC reference number: 2733564

General Medical Council.

GENERAL MEDICAL COUNCIL (2010b) Good practice in research and Consent to research

GLASZIOU, P. \& CHALMERS, I. (2004) Ethics review roulette: what can we learn? Bmj, $328,121-2$.

HAFFERTY, F. W. \& FRANKS, R. (1994a) The Hidden Curriculum, Ethics Teaching, and the Structure of Medical Education. Acad Med, 69, 861871.

HAFFERTY, F. W. \& FRANKS, R. (1994b) The Hidden Curriculum, Ethics Teaching, and the Structure of Medical Education. Academic Medicine, 69, 861.

HEITMAN, E., OLSEN, C. H., ANESTIDOU, L. \& BULGER, R. E. (2007a) New graduate students' baseline knowledge of the responsible conduct of research. Acad Med, 82, 838-45.

HEITMAN, E., OLSEN, C. H., ANESTIDOU, L. \& BULGER, R. E. (2007b) New Graduate Students' Baseline Knowledge of the Responsible Conduct of Research. Academic Medicine, 82, 838-845.

INFORMA HEALTHCARE (2010) Resources for Authors. Iondon, Informa plc.

JAMROZIK, K. (2004) Research ethics paperwork: what is the plot we seem to have lost? British Medical Journal, 286-287.

KALICHMAN, M. W. (2007a) Responding to Challenges in Educating for the Responsible Conduct of Research. Acad Med

\section{2, 870-875.}

KALICHMAN, M. W. (2007b) Responding to Challenges in Educating for the Responsible Conduct of Research. Academic Medicine, 82, 870-875.

KALICHMAN, M. W. \& PLEMMONS, D. K. (2007) Reported Goals for Responsible Conduct of Research Courses. Academic Medicine, 82, 846-852 10.1097/ACM.0b013e31812f78bf.

LEHMANN, L. S., KASOFF, W. S., KOCH, P. \& FEDERMAN, D. D. (2004) A Survey of Medical Ethics Education at U.S. and Canadian Medical Schools. Academic Medicine, 79, 682-689. 
MCGEE, R., ALMQUIST, J., KELLER, J. L. \& JACOBSEN, S. J. (2008) Teaching and learning responsible research conduct: influences of prior experiences on acceptance of new ideas. Account Res, 15, 30-62.

MILES, S. H., LANE, L. W., BICKEL, J., WALKER, R. M. \& CASSEL, C. K. (1989) Medical ethics education: coming of age

Acad Med

64, 705-714.

PLEMMONS, D. K. \& KALICHMAN, M. W. (2007) Reported Goals for Knowledge to be Learned in Responsible Conduct of Research Courses. Journal of Empirical Research on Human Research Ethics, 2, 57-66.

POWELL, S. T., ALLISON, M. A. \& KALICHMAN, M. W. (2007) Effectiveness of a responsible conduct of research course: a preliminary study. Science and Engineering Ethics, 13, 249-264.

ROBINSON, L., DREWERY, S., ELLERSHAW, J., SMITH, J., WHITTLE, S. \& MURDOCH EATON, D. (2007) Research governance: impeding both research and teaching? A survey of impact on undergraduate research opportunities. Med Educ, 41, 729-36.

SPONHOLZ, G. (2000) Teaching scientific integrity and research ethics. Forensic Science International, 113, 511-514.

STENECK, N. H. (2007) Introduction to the Responsible Conduct of Research. Washington DC, U.S. Department of Health and Human Services.

STENECK, N. H. \& BULGER, R. E. (2007) The history, purpose, and future of instruction in the responsible conduct of research. Acad Med, 82, 829-34.

THE ROYAL COLLEGE OF PHYSICIANS AND SURGEONS OF CANADA (2005) CanMEDS 2005 Framework.

WAKEFIELD, A. J., TAYLOR, B. \& ROBERTS, G. T. (1999) MMR vaccination and autism. Lancet, 949-950.

WALD, D. S. (2004) Bureaucracy of ethics applications. British Medical Journal, 282-284. 